\title{
Prevalencia de anticuerpos para Trypanosoma cruzi en caninos de dos municipios endémicos de Boyacá
}

\section{Prevalence of antibodies for Trypanosoma cruzi in canines from two endemic municipalities of Boyacá}

\author{
Diego Manrique-Abril, ${ }^{1}$ MVZ, Fred Manrique-Abril,1,2* Ph.D, Myriam Lorca H, ${ }^{3}$ Ph.D, \\ Juan Ospina D, ${ }^{1}$ M.Sc.
}

${ }^{1}$ Universidad Pedagógica y Tecnológica de Colombia Tunja. Grupo de Investigación en Salud Pública. Tunja, Boyacá, Colombia. ${ }^{2}$ Universidad Nacional de Colombia. Bogotá D.C. Colombia. ${ }^{3}$ Consultora OMS, Director científico CAMPVS, Universidad de Chile. Santiago. Chile. *Correspondencia: fgma75@ hotmail.com

Recibido: Agosto de 2010; Aceptado: Junio de 2011.

\section{RESÚMEN}

Objetivo. evaluar la prevalencia de anticuerpos anti Trypanosoma cruzi ( $T$. cruzi) en una muestra de caninos domésticos residentes en dos municipios endémicos. Materiales y métodos. Se tomaron muestras séricas de 20 caninos procedentes de hogares donde residen mujeres gestantes seropositivas y 40 perros habitantes de hogares de mujeres gestantes seronegativas en Miraflores y Moniquira, Boyacá. El análisis se realizó mediante prueba diagnóstica rápida dipstick de InBios. Resultados. Se encontró prevalencia del $16.7 \%$ en Moniquirá y del 13.3\% Miraflores respectivamente con una prevalencia general del $15 \%$ en los dos municipios. Se halló riesgo 3 veces mayor de que ocurra la infección en caninos, en los hogares donde residen gestantes seropositivas; además la infestación por pulgas y garrapatas en el animal, hábitat cercano a la vivienda, se relacionan con mayor seropositividad en el canino. Conclusiones. La raza, el sexo, la presencia de aves en la casa y al examen clínico general son considerados factores pronósticos en en la infección por Trypanosoma cruzi en caninos. Como factores protectores se identificó la desparasitación y vacunación de los animales.

Palabras clave: Colombia, enfermedad de chagas, infección, perros (Fuente: $C A B)$. 


\begin{abstract}
Objective. To assess the prevalence of antibodies to Trypanosoma cruzi ( $T$. cruzi) in a sample of domestic dogs living in two endemic municipalities. Materials and methods. Serum samples were taken from 20 dogs from households with seropositive pregnant women, and 40 dogs from households with seronegative pregnant women in Miraflores and Moniquirá, Boyacá. The analysis was carried out through INBios RDT dipstick. Results. A prevalence of $16.7 \%$ and $13.3 \%$ in Moniquirá and Miraflores respectively was found, with an overall prevalence of $15 \%$ in the two municipalities. There is a risk of acquiring infection in dogs three times greater in households with sero positive pregnant women. Flea and tick infestation in the animal, and habitat near the house, were associated with higher prevalence in the canine. Conclusions. Breed, gender, presence of birds in the house and clinical examination are considered prognostic factors for the infection by Trypanosoma cruzi in canines. As protection factors de-worming and vaccination of animals were identified.
\end{abstract}

Key words: Colombia, chagas disease, dogs, infection (Source: $C A B$ ).

\title{
INTRODUCCIÓN
}

La enfermedad de Chagas es una zoonosis causada por Trypanosoma cruzi, parásito protozoario flagelado que se localiza exclusivamente en el continente americano. (1)

La Organización Panamericana de la Salud (OPS) ha estimado que de los 360 millones de personas que viven en países endémicos, 90 millones corren el peligro de contraerla y de 16 a 18 millones ya están infectados, y en un futuro podrían sufrir daños cardiacos, gastrointestinales y neurológicos; es así que la enfermedad de Chagas es considerada como la cuarta causa de mortalidad en América Latina, provocando unas 43000 muertes cada año (2).

Los perros están presentes en la familia Boyacense como compañía y guarda en los hogares, especialmente en las zonas rurales, pues se encuentran en el interior de las viviendas en altas proporciones, de hasta $76 \%$ de hogares (3), aunque las condiciones de higiene y cuidado de la mascota son sustancialmente diferentes, dado el estilo de vida y cultura de los habitantes de zonas urbanas.

La convivencia con caninos representa significativos riesgos en la medida que las condiciones ambientales, particularmente en zonas endémicas favorecen múltiples infestaciones tanto por insectos vectores como por parásitos, lo que disminuye en el caso de la enfermedad de Chagas, la eficacia de las acciones de rociado con insecticidas al interior de las casas como medida preventiva de control de los vectores.

Los riesgos derivados de la convivencia con perros han sido ampliamente documentados, y ya se les ha identificado y reconoce como reservorios de enfermedades que pueden ser trasmitidas al hombre como la enfermedad de Chagas (4), leptospirosis, leishmaniosis, rabia, tuberculosis y otras. Los Perros domésticos desempeñan un papel importante en la epidemiología de la enfermedad de Chagas siendo portadores asintomáticos y de alta parasitemia en esta infección (5).

En Boyacá a pesar de tener zonas endémica de la enfermedad solo se encuentra un estudio adelantado en Soatá y Berbeo, donde se demostró que $10.72 \%$ de perros domésticos estaban infectados por $T$. cruzi identificados mediante inunofluoresencia indirecta en una muestra de 261 perros (6). De la misma manera, en el área de Miraflores y Moniquira Boyaca un estudio de determinación de enfermedad de Chagas transmitida por vía transplacentaria reportó una prevalencia de infección en mujeres gestantes, de $33.28 \%$ (22 muestras positivas de 661), una prevalencia muy alta en América Latina. También se reportó por primera vez una caso de Chagas congénito; el $T$. cruzi involucrado fue de Linaje tipo I y II (7-11).

En el marco de los programas institucionales de control vectorial que se adelantan en Colombia, también se ha identificado como un importante factor de riesgo la tenencia de mascotas, estudios realizados en diferentes regiones de Colombia han demostrado seroprevalencias en perros superiores al $27 \%$, siendo estos animales con frecuencia, víctimas de la infección y lo más importante portadores de altas parasitemias, lo que mantiene una fuente de mantención y amplificación de la infección para los vectores cercanos al animal $(6,7,12)$.

En humanos, se considera que la transmisión congénita es frecuente en lugares con alta prevalencia de la enfermedad. Sin embargo, 
aún no se han adelantado estudios que permitan establecer si este mecanismo de transmisión también puede ocurrir en perros y si constituye un mecanismo amplificador de la infección y de mayor riesgo de infección para los vectores.

En América latina, existe evidencia que demuestra elevadas prevalencias de enfermedad de Chagas en humanos, asociada con tenencia de reservorios animales caninos en sus casas $(4,6,13)$.

El objetivo del presente estudio fue determinar, en una muestra aleatoria, la frecuencia de ocurrencia de infección por $T$. cruzi en perros, evaluar factores de riesgo ambientales y de la vivienda y explorar posibles asociaciones correlacionadas con la prevalencia de la infección en humanos, y condiciones del habitat que podrían favorecer la enfermedad de chagas.

\section{MATERIALES Y MÉTODOS}

Tipo de estudio. Estudio de prevalencia analítica, En primer lugar se analiza como un estudio epidemiológico descriptivo y en un segundo momento se explora la fuerza de asociación entre diferentes variables de riesgo con factores que podrían interpretarse causalmente como asociados a la probabilidad del contagio, en un análisis bivariado y estratificado.

Tamaño de muestra. El tamaño de la muestra fue calculado con base en la proporción de infestación reportada en otros estudios similares utilizando un nivel de significancia del $95 \%$ y una precisión del $2.5 \%$. Así el tamaño de la muestra requerido fue de 60 individuos, los cuales se consideraron individualmente como unidades de observación y análisis del estudio.

\begin{abstract}
Selección de participantes y área geográfica. El estudio se realizo luego de conocer un estudio que determinó la prevalencia de enfermedad de Chagas en mujeres gestantes, financiado por Colciencias; a partir de la base de datos inicial, se seleccionaron veinte 20 caninos que residían como mascotas en hogares donde al menos una mujer gestante había sido encontrada positiva en el suero para $T$. Cruzi. Adicionalmente, fue evaluado un grupo control conformado por 40 perros residentes en hogares de mujeres gestantes que fueron reportadas como seronegativas a la enfermedad de Chagas, en los municipios de Miraflores y Moniquirá Boyacá.
\end{abstract}

Técnicas usadas. Para la determinación de la infestación mediante tamizaje se utilizó una prueba rápida para detectar anticuerpos contra $T$. cruzi, agente causal de la enfermedad de Chagas: RDT (Trypanosoma Detect, INBIOS InternationalTM, Seattle, USA)(14). La prueba está basada en un antígeno recombinante multiepitope mezclado con una solución de oro. El antígeno recombinante incluye las moléculas ITC-6 e ITC-8.2 derivados de diferentes antigenos de $T$. cruzi, inclusive los péptidos 2, TCD, TcF, TCLo, y SAPA.

El procedimiento se efectuó de acuerdo a las instrucciones de la empresa fabricante. Entre 10 y $20 \mu \mathrm{l}$. de suero canino fueron dispuestos sobre la tira del "dipstick" y posteriormente se agregaron 3 gotas de la solución buffer reveladora, provista en el Kit. Después de 10 minutos se observó la línea de control rojo. Si el resultado fue positivo apareció una segunda línea por debajo del control en el campo donde se ubicó el antígeno. El reactivo INBIOS ha demostrado tener una sensibilidad de $99.3 \%$ con $0.01 \%$ de falsos positivos sobre el total de casos estudiados y una especificidad de $99.3 \%$ con un total de 0.7 de falsos negativos sobre el total de muestras, en un estudio doble ciego, en el que primero se estudiaron muestras de suero analizadas previamente con ELISA e Inmunofluorescencia indirecta (IFI), y a continuación con el RDT (14).

También se aplicó, previo consentimiento informado, a las personas residentes en estos hogares, una encuesta que exploraba aspectos demográficos, sanitarios y etológicos de los animales para evaluar posibles relaciones con la infección.

Análisis de datos. El análisis estadístico

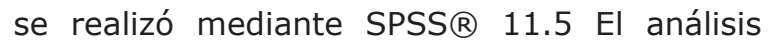
exploratorio evaluó frecuencias, test de ANOVA para comparación de grupos y para evaluar la asociación de variables se realizaron pruebas de $\chi^{2}$ y Test exacto de Fisher, se establecieron prevalencias y como medida del efecto, razones de prevalencia con sus correspondientes intervalos de confianza al $95 \%$.

\section{RESULTADOS}

Se encontró una prevalencia del 16.7\% en Moniquirá y del $13.3 \%$ Miraflores respectivamente, con una prevalencia global de $15 \%$ en los dos municipios (Tabla 1 ).

La distribución de seropositividad es mayor en animales criollos y de hábitat intradomiciliar, se observó mayor frecuencia de seropositividad para 
Tabla 1. Prevalencia de anticuerpos para Chagas en caninos procedentes del domicilio de maternas con y sin infección por T. cruzi de Miraflores y Moniquirá, 2009. Análisis estratificado.

\begin{tabular}{|c|c|c|c|c|c|c|}
\hline & \multirow{2}{*}{ Variables } & \multicolumn{2}{|c|}{ Caninos Positivos } & \multicolumn{2}{|c|}{ Caninos Negativos } & \multirow{2}{*}{ TOTAL } \\
\hline & & $\mathbf{N}$ & $\%$ & $\mathbf{N}$ & $\%$ & \\
\hline \multirow[t]{2}{*}{ Municipio } & Miraflores & 4 & $13.3 \%$ & 26 & $86.7 \%$ & 30 \\
\hline & Moniquirá & 5 & $16.7 \%$ & 25 & $83.3 \%$ & 30 \\
\hline \multirow[t]{8}{*}{ Raza } & Cocker & 0 & $.0 \%$ & 1 & $100.0 \%$ & 1 \\
\hline & Fresh & 0 & $.0 \%$ & 5 & $100.0 \%$ & 5 \\
\hline & Labrador & 0 & $.0 \%$ & 1 & $100.0 \%$ & 1 \\
\hline & Mastín & 0 & $.0 \%$ & 1 & $100.0 \%$ & 1 \\
\hline & Mastín X Criollo & 0 & $.0 \%$ & 1 & $100.0 \%$ & 1 \\
\hline & Pitbull $\times$ Criollo & 0 & $.0 \%$ & 1 & $100.0 \%$ & 1 \\
\hline & Rotweiller & 0 & $.0 \%$ & 1 & $100.0 \%$ & 1 \\
\hline & Rotw $\times$ Pastor & 0 & $.0 \%$ & 1 & $100.0 \%$ & 1 \\
\hline \multirow[t]{2}{*}{ Sexo } & Hembra & 3 & $16.7 \%$ & 15 & $83.3 \%$ & 18 \\
\hline & Macho & 6 & $14.3 \%$ & 36 & $85.7 \%$ & 42 \\
\hline \multirow[t]{3}{*}{ Hábitat } & Intradomiciliar (incluye dormitorios) & 3 & $27.3 \%$ & 8 & $72.7 \%$ & 11 \\
\hline & Domiciliar & 2 & $11.8 \%$ & 15 & $88.2 \%$ & 17 \\
\hline & Peridomiciliar & 4 & $12.5 \%$ & 28 & $87.5 \%$ & 32 \\
\hline
\end{tabular}

Tabla 2. Factores de riesgo-protectores probablemente asociados a la prevalencia de enfermedad de Chagas en caninos. Miraflores y Moniquirá, 2009.

\begin{tabular}{|c|c|c|c|c|c|c|c|}
\hline \multicolumn{2}{|c|}{ Variable } & \multirow{2}{*}{$\frac{\text { Caninos positivos }}{5}$} & \multirow{2}{*}{$\begin{array}{c}\text { Caninos negativos } \\
25\end{array}$} & \multirow{3}{*}{$\frac{\mathrm{RP}}{1.25}$} & \multicolumn{2}{|c|}{$\begin{array}{l}\text { Asociación } \\
\text { IC } 95 \%\end{array}$} & \multirow{3}{*}{$\frac{\chi^{2}}{0.71}$} \\
\hline \multirow[t]{2}{*}{ Municipio } & Moniquirá & & & & & & \\
\hline & Miraflores & 4 & 26 & & 0.31 & 4.21 & \\
\hline \multirow[t]{2}{*}{ Materna en la vivienda } & Positivo & 5 & 15 & \multirow{2}{*}{3.00} & \multirow{2}{*}{0.71} & \multirow{2}{*}{12.74} & \multirow{2}{*}{0.13} \\
\hline & Negativo & 4 & 36 & & & & \\
\hline \multirow[t]{2}{*}{ Sexo } & Hembra & 3 & 15 & \multirow{2}{*}{1.20} & \multirow{2}{*}{0.26} & \multirow{2}{*}{5.44} & \multirow{2}{*}{0.81} \\
\hline & Macho & 6 & 36 & & & & \\
\hline \multirow[t]{2}{*}{ Raza } & Criollo & 9 & 29 & \multirow{2}{*}{$14.49 *$} & \multirow{2}{*}{0.80} & \multirow{2}{*}{262.38} & \multirow{2}{*}{0.05} \\
\hline & Otra & 0 & 22 & & & & \\
\hline \multirow[t]{2}{*}{ Domicilio } & Intra domicilio & 5 & 23 & \multirow{2}{*}{1.52} & \multirow{2}{*}{0.37} & \multirow{2}{*}{6.33} & \multirow{2}{*}{0.56} \\
\hline & Periferia & 4 & 28 & & & & \\
\hline \multirow[t]{2}{*}{ Desparasitado } & $\mathrm{Si}$ & 4 & 40 & \multirow{2}{*}{0.22} & \multirow{2}{*}{0.05} & \multirow{2}{*}{0.96} & \multirow{2}{*}{0.03} \\
\hline & No & 5 & 11 & & & & \\
\hline Vacunado & $\mathrm{Si}$ & 8 & 49 & 033 & 0.03 & 4.03 & 0.36 \\
\hline & No & 1 & 2 & 0.33 & & & \\
\hline Presencia De Pulgas & Si & 8 & 25 & 832 & 097 & 7144 & 003 \\
\hline & No & 1 & 26 & 8.32 & 0.97 & 11.44 & 0.03 \\
\hline Presencia De Garrapatas & $\mathrm{Si}$ & 4 & 12 & & & & \\
\hline & No & 5 & 39 & 2.60 & 0.60 & 11.26 & 0.19 \\
\hline Presencia De Gatos & $\mathrm{Si}$ & 4 & 25 & & & & \\
\hline & No & 5 & 26 & 0.83 & 0.20 & 3.46 & 0.80 \\
\hline Presencia De Aves & $\mathrm{Si}$ & 9 & 35 & $883 *$ & & 1610 & 80 0 \\
\hline & No & 0 & 16 & $8.83^{*}$ & 0.48 & 161.0 & 0.08 \\
\hline Vida Nocturna & Si & 6 & 24 & 2.25 & 0.51 & 999 & 0.28 \\
\hline & No & 3 & 27 & 2.25 & 0.51 & 9.99 & 0.28 \\
\hline Estado Clinico & Caquéctico & 5 & 3 & & & & \\
\hline & Normal & 4 & 48 & 20 & 3.45 & 115.97 & 0.00 \\
\hline
\end{tabular}

Rp: Razón de prevalencias. Ic95\%: Intervalo de confianza Al 95\%. $\chi^{2}$ : P Valor de Chi Cuadrado. *: Con corrección de Yates. 
el animal, en las viviendas construidas con adobe no cocido.

Los resultados del análisis bivariado, si bien no fueron concluyentes, sugieren que en los hogares con gestantes seropositivas, es 3 veces mayor la probabilidad de encontrar la infección en caninos y viceversa. Se encontró asociación positiva con significancia estadística para la raza, la presencia de pulgas y el estado clínico. ( $\mathrm{p} \leq 0.05$, Tabla 2).

La desparasitación como medida sanitaria en los animales, se halló como factor protector para el evento seropositividad para $T$. cruzi con significancia $(p \leq 0.05)$.

El sexo, la presencia de aves y el examen clínico general, en particular la frecuencia respiratoria $(p=0.0048)$ y la condición clínica $(p=0.0004)$, podrían ser considerados factores pronósticos en la aproximación al diagnóstico de infección por Chagas canino, especialmente en áreas geográficas endémicas (Tabla 3 ).

Tabla 3. Variables cuantitativas clínicas por resultado de seropositividad de enfermedad de Chagas en caninos $(\bar{X} ; \pm)$.

\begin{tabular}{ccccccc}
\hline Resultado & EDAD & DISTAN & FR & FC & CC & T \\
\hline \multirow{2}{*}{ Positivo } & 34.4 & 3.4 & 34.2 & 87.6 & 2.7 & 38.2 \\
& \pm 24.57 & \pm 3.0 & \pm 4.9 & \pm 5.8 & \pm 0.7 & \pm 0.75 \\
\multirow{2}{*}{ Negativo } & 43.5 & 3.6 & 30.6 & 86.5 & 3.3 & 37.75 \\
& 30.14 & \pm 2.4 & \pm 5.6 & \pm 7.4 & \pm 0.5 & \pm 0.65 \\
\hline \multirow{2}{*}{ Total } & 42.1 & 3.6 & 31.1 & 86.6 & 3.2 & 37.81 \\
& \pm 29.36 & \pm 2.4 & \pm 5.6 & \pm 7.2 & \pm 0.6 & \pm 0.68 \\
\hline
\end{tabular}

$\mathrm{FR}=$ Frecuencia respiratoria; $\mathrm{FC}=$ Frecuencia cardiaca; $\mathrm{CC}=$ condición corporal; $\mathrm{T}=$ temperatura ${ }^{\circ} \mathrm{C} ; \mathrm{De}=$ Desviación estándar.

Las variables de condición fisiológica, medidas en los animales no presentaron cambios significativos entre los positivos y negativos, esto tiene relevancia ya que la enfermedad se presenta silenciosa sin signos patognomónicos.

\section{DISCUSIÓN}

La prevalencia estimada es similar a lo reportado en Argentina por Gurtler (13,1518) y en Colombia por Gómez (6); aunque la literatura muestra cifras variables, probablemente relacionadas con la intensidad de la endemia y la técnica empleada, los reportes muestran diferencias significativas en prevalencia que van desde $1.4 \%$ en Tolima; en Estados Unidos: Texas $20.3 \%$ y Luisiana $4.7 \% ; 27.7 \%$ en Costa Rica; $41.2 \%$ en Argentina; $17.5 \%$ en México (19).
La seropositividad encontrada en los caninos examinados es una señal de alerta para las autoridades sanitarias de las zonas endémicas en el departamento de Boyacá, particularmente en los encargados de los programas de control de zoonosis.

La sectorización y los factores asociados que se evidencian en el entorno de la mayoría de los resultados positivos invita a profundizar investigaciones epidemiológicas más detalladas en las poblaciones de caninos domésticos en la localidad, en particular si se considera el papel preponderante que juegan los factores socioeconómicos como aspectos determinantes que podrían incidir significativamente en la dispersión de los contagios de esta enfermedad. Además, para los ámbitos académicos, estudios como el presente destacan la importancia de capacitar a los médicos veterinarios en las técnicas de control sanitario y vigilancia epidemiológica en la perspectiva de su participación en los programas de control de enfermedades zoonóticas.

Se hace necesaria la implementación de programas de tamizaje en zonas endémicas tanto en población canina como humana y conocer la magnitud del problema realmente, ya que los mapas se basan en la infestación de vectores y se extrapola sin considerar realmente la infección.

La información y el reconocimiento del ciclo biológico de $T$. cruzi debe ser prioridad en los planes de salud publica. Educar, comunicar e informar sobre la enfermedad debe ser política prioritaria en esta zona. Esto puede reducir de manera importante la transmisión a los humanos (20).

La prevalencia hallada en el presente estudio confirma el papel que juegan los perros domésticos en el proceso de infección humana al identificarlo como reservorio de T. cruzi, y de su importancia en el sostenimiento del ciclo del parásito para el desarrollo de la infección en humanos.

La presencia de mascotas caninas infectadas reviste profundo valor sanitario dada la proximidad de estos animales al entorno humano, sobre todo si se tiene en cuenta que los triatomíneos, aun siendo más afines a alimentarse con sangre humana no desechan la posibilidad de hacerlo a expensas de la sangre de otros mamíferos de sangre caliente, como se comprueba en estudios adelantados en Centro América en los que se reporta la presencia de sangre de perros en hasta un $50 \%$ de triatomíneos capturados y examinados (21). 
En relación con la variable sexo, llama la atención el hecho de que en otros estudios se ha encontrado un mayor porcentaje de infestación en los machos, diferencia que puede ser 3 ó 4 veces mayor, circunstancia que se explicaría por la mayor movilidad de los machos, lo que los expondría más al contacto con los triatomíneos en áreas peridomiciliarias y boscosas en los asentamientos rurales.

Desde la perspectiva clínica, el hallazgo de seropositividad puede constituir un primer paso en la exploración de las manifestaciones de enfermedad cardiaca causada por T. Cruzi en los perros, habida cuenta de la asociación encontrada con la caquexia y aumento de la frecuencia respiratoria, hallazgos que en cierta medida coinciden con lo reportado respecto del estrés respiratorio como manifestación de la enfermedad en caninos (22).

Una premisa importante que se desprende de los resultados del estudio tiene que ver con la necesidad de implementar estrategias para contrarrestar la asociación entre vector y perros (20), de los municipios de Moniquirá y Miraflores, puesto que en los lugares donde se han adelantado investigaciones, se ha encontrado una correlación significativa entre el mayor volumen de canes infectados y el número de personas enfermas. En este sentido se considera que el perro es un agente amplificador, es decir, es un mamífero que convive o habita en cercanía con los seres humanos, no tiene capacidad para evitar la picadura de los triatomíneos a la vez que estos mismos podrían picar al ser humano en el mismo hábitat, o en uno muy próximo, circunstancia que otorga prioridad epidemiológica a los perros respecto a otros animales silvestres $(13,15-18,20)$.

La circunstancia arriba descrita se corrobora con la fuerte asociación observada entre la presencia de mujeres gestantes infectadas y perros seropositivos en la misma vivienda, condición descrita con anterioridad por el equipo de investigación de salud pública de la Universidad Pedagógica y Tecnológica de Colombia (UPTC) $(7,20,23)$, relación también documentada en estudios latinoamericanos $(4,5,8,13,17,24)$.

Desde la perspectiva de las medidas sanitarias de control y prevención esta relación reviste importancia en otra que tiene que ver con la probabilidad de que la presencia de mamíferos en o alrededor de las viviendas favorecería la proliferación de vectores triatomíneos por constituir una fuente importante de alimentación para ellos, aumentando en la infestación de las habitaciones cuando hay presencia canina alrededor de la vivienda $(4,6,12,18,25)$.

Si bien la transmisión transplacentaria de la tripanosomiasis en hembras de caninos no se ha documentado de manera concluyente, las similitudes descritas en los cuadros clínicos de la enfermedad en caninos y humanos invitan a proponer el estudio de la transmisión congénita en perros, teniendo en cuenta que la enfermedad por vía transplacentaria está cobrando significativa importancia en relación con el contagio vectorial en algunas zonas, por lo cual la transmisión vertical generacional se convertiría en el elemento a controlar tanto en reservorios como en humanos (7-11,26).

En conclusión, se documentan altas prevalencias de infección en caninos, que se correlacionan significativamente con las infecciones en mujeres embarazadas, hallazgo que constituye una alerta epidemiológica para emprender medidas de control y prevención mediante tamizajes intensivos y educación a las comunidades sobre los riesgos de contagio que se incrementan para las personas que conviven con mascotas caninas en franca cercanía, debido a que los vectores tienen una fuente de alimentación auxiliar importante en periodos en que no hay población humana presente.

La presencia de caninos infectados aumenta el riesgo de transmisión a los vectores, y de esta manera la probabilidad de infección en los humanos. Los perros podrían resultar buenos centinelas en los procesos de vigilancia en los programas de control vectorial.

\section{Agradecimientos}

A los laboratorios CAMPVS e InBios por los aportes en el campo científico y logístico en el desarrollo del presente estudio. A los integrantes del grupo de investigación en salud pública (GISP) por el apoyo académico y estructural, a los estudiantes que colaboraron en la recolección de las muestras y captura de datos. A las familias que visitamos por abrir sus puertas y brindarnos su valioso tiempo. 


\section{REFERENCIAS}

1. Schofield CJ, Jannin J, Salvatella R. The future of Chagas disease control. Trends Parasitol 2006;22(12):583-588.

2. Organización Mundial de la Salud, Salud. OPdl. Estimación cuantitativa de la Enfermedad de Chagas en las Americas. Geneva: World Health Organization 2006.

3. Ospina JM, Manrique-Abril FG, Ariza NE. Salud, ambiente y trabajo en poblaciones vulnerables: los cultivadores de papa en el centro de Boyacá. Rev Fac Nac Salud Pública 2009;26(2):143-152.

4. Castanera MB, Lauricella MA, Chuit R, Gurtler RE. Evaluation of dogs as sentinels of the transmission of Trypanosoma cruzi in a rural area of north-western Argentina. Ann Trop Med Parasitol 1998;92(6):670-682.

5. Bradley KK, Bergman DK, Woods JP, Crutcher JM, Kirchhoff LV. Prevalence of American trypanosomiasis (Chagas disease) among dogs in Oklahoma. J Am Vet Med Assoc 2000;217(12):1853-1857.

6. Gómez BCT, Vallejo GA, Guhl F. Seroprevalencia de trypanosoma cruzi en perros de dos áreas endémicas de Colombia. Rev Fac Med 2008;16(1):2-18.

7. Manrique-Abril FG, Herrera-Amaya GM, Ospina Díaz JM, Pavia PX, Puerta $C J$, Montilla $M$, et al. Prevalencia de Enfermedad de Chagas en maternas e incidencia transplacentaria en Boyacá. Biomedica 2009;29(supl 1):315-316.

8. Manrique-Abril FG, Ospina Díaz JM, HerreraAmaya G, Nicholls RS, Puerta C, Florez AC, et al. P093. Congential Chagas disease in Miraflores and Moniquira Boyacá-Colombia. Bol Mal Salud Amb 2007;42(1):238-239.

9. Pavia $P$, Montilla $M$, Nicholls $R S$, Florez AC, Manrique-Abril FG, Ospina Díaz JM, et al. Análisis de un caso de enfermedad de chagas transplacentario en Moniquira (Boyacá) mediante AP-PCR. Biomedica 2007;27(supl 2):238-239.
10. Pavia PX, Montilla M, Florez AC, HerreraAmaya GM, Ospina Díaz JM, Manrique-Abril FG, et al. Caracterización molecular de 9 sepas de Trypanosoma cruzi aisladas de casos de Chagas congénito en los municipios de Miraflores y Moniquirá, Boyacá. Biomedica 2009; 29(supl 1):180.

11. Pavia PX, Montilla M, Florez AC, HerreraAmaya GM, Ospina Díaz JM, ManriqueAbril FG, et al. Reporte del primer caso de enfermedad de Chagas transplacentaria analizado por AP-PCR en Moniquirá, Boyacá. Biomedica 2009; 29(4):513-522.

12. Campbell-Lendrum DH, Angulo VM, Esteban L, Tarazona Z, Parra GJ, Restrepo M, et al. House-level risk factors for triatomine infestation in Colombia. Int J Epidemiol 2007; 36(4):866-872.

13. Gurtler RE, Cecere MC, Lauricella MA, Cardinal MV, Kitron U, Cohen JE. Domestic dogs and cats as sources of Trypanosoma cruzi infection in rural northwestern Argentina. Parasitology 2006;134(1):69-82.

14. Lorca M, Contreras M, Salinas P, Guerra A, Raychaudhuri S. Evaluation of a rapid diagnosis test for trypanosoma cruzi infection in serum sample. Parasitol Latinoam 2008;63:29-33.

15. Gurtler RE, Cecere MC, Castanera MB, Canale D, Lauricella MA, Chuit R, et al. Probability of infection with Trypanosoma cruzi of the vector Triatoma infestans fed on infected humans and dogs in northwest Argentina. Am J Trop Med Hyg 1996;55(1):24-31.

16. Gurtler RE, Cecere MC, Rubel DN, Schweigmann NJ. Determinants of the domiciliary density of Triatoma infestans, vector of Chagas disease. Med Vet Entomol 1992;6(1):75-83.

17. Gurtler RE, Chuit R, Cecere MC, Castanera MB, Cohen JE, Segura EL. Household prevalence of seropositivity for Trypanosoma cruzi in three rural villages in northwest Argentina: environmental, demographic, and entomologic associations. Am J Trop Med Hyg 1998;59(5):741-749. 
18. Gurtler RE, Cohen JE, Cecere MC, Lauricella MA, Chuit R, Segura EL. Influence of humans and domestic animals on the household prevalence of Trypanosoma cruzi in Triatoma infestans populations in northwest Argentina. Am J Trop Med Hyg 1998;58(6):748-758.

19. Romero-Peñuela MH, Sánchez-Valencia JA. Seroprevalencia de Trypanosoma cruzi por la técnica de Western Blot en población canina del Departamento del Tolima, Colombia. Vet Zootec 2008;2(2):48-52.

20. Cohen JE, Gurtler RE. Modeling household transmission of American trypanosomiasis. Science 2001;293(5530):694-698.

21. Calderón-Arguedas O, Chinchilla M, Garcia $F$, Vargas M. Preferencias alimentarias de Triatoma dimidiata (Hemiptera: Reduviidae) procedentes de la Meseta Central de Costa Rica a finales del siglo XX. Parasitol Día $2001 ; 25(3-4): 78-81$.

22. Barr SC, Simpsom RM, Schmidt SP, Bunge MM, Authement JM, Lozano F. Chronic dilatative myocarditis caused by Trypanosoma cruzi in two dogs. J Am Vet Med Assoc 1989; 1;195(9):1237-1241.
23. Camacho Daza SM, Saavedra Infante $D L$, Manrique-Abril FG. Caracterización de las prácticas de autocuidado de las gestantes en riesgo de adquirir enfermedad de Chagas en Miraflores y Moniquirá Boyacá. Tunja: Biblioteca Lascasas, 2007; 3(3). URL Disponible en http://www.index-f. com/lascasas/documentos/lc0259.php.

24. World Health Organization. Control of Chagas Disease: Second Report of a WHO Expert Committee. Geneva: World Health Organization; 2002. URL Disponible en: http://whqlibdoc.who.int/trs/WHO_ TRS_905.pdf.

25. Guhl F, Vallejo GA. Interruption of Chagas disease transmission in the Andean countries: Colombia. Mem I Oswaldo Cruz 1999; 94(Suppl 1):413-415.

26. Manrique-Abril FG, Ospina Díaz JM, HerreraAmaya GM, Nicholls RS, Montilla M, Florez AC, et al. Enfermedad de chagas trasplacentaria en Miraflores y Moniquira, Boyacá. Biomedica 2007;27(supl 2):172-173. 